\title{
Effects of Temperature and Humidity on Radio Signal Strength in Outdoor Wireless Sensor Networks
}

\author{
Jari Luomala and Ismo Hakala \\ University of Jyvaskyla, Kokkola University Consortium Chydenius \\ P.O. Box 567, FI-67701 Kokkola, Finland \\ Email: \{jari.luomala, ismo.hakala\}@chydenius.fi
}

\begin{abstract}
Many wireless sensor networks operating outdoors are exposed to changing weather conditions, which may cause severe degradation in system performance. Therefore, it is essential to explore the factors affecting radio link quality in order to mitigate their impact and to adapt to varying conditions. In this paper, we study the effects of temperature and humidity on radio signal strength in outdoor wireless sensor networks. Experimental measurements were performed using Atmel ZigBit $2.4 \mathrm{GHz}$ wireless modules, both in summer and wintertime. We employed all the radio channels specified by IEEE 802.15.4 for 2.4GHz ISM frequency band with two transmit power levels. The results show that changes in weather conditions affect received signal strength. Of the studied weather variables, variation in signal strength can be best explained by the variation in temperature. We also show that frequency diversity can reduce the effects of channel-specific variation, and the difference between the transmit power levels.
\end{abstract}

\section{INTRODUCTION}

$\mathbf{M}$ ANY wireless sensor networks (WSNs) and their applications are in use outdoors exposed to changing environmental conditions. Weather conditions particularly can have a significant impact on the performance of WSNs and therefore cannot be ignored [1], [2], [3]. While the location of nodes may be fixed and their surroundings might remain almost static, the weather will not remain stable. The ambient temperature and humidity will change and fluctuate temporally, having both diurnal and seasonal variation. In addition, there can be spatial variation in weather, which affects WSN due to microclimates. While changes in weather conditions are inevitable and may have significant effects, they are usually measurable and could be mitigated based on experimental measurements. Hence, it is essential to explore weather-related factors affecting radio link quality in order to mitigate their impact and to adapt to varying conditions.

The effects of weather conditions on link quality (e.g., signal strength) in WSNs have been explored in quite a few studies (e.g., [2], [4], [3], [1], [5], [6], [7], [8], [9]). However, no clear consensus has been achieved so far. Some studies report that temperature is the dominating factor affecting signal strength while others claim that humidity is the main reason. Some suggest also other reasons. Furthermore, research methods, radios and platforms employed and a number of explored weather variables vary between studies, occasionally resulting in contradictory results and conclusions. Hence, there definitively seems to be a need for further studies.

This paper sets out to find out the effects of temperature and humidity on radio signal strength in outdoor WSNs. Experimental measurements were carried out using Atmel ZigBit $2.4 \mathrm{GHz}$ wireless modules [10] with AT86RF230 radios [11] in a university campus area during December 2013 and July 2014. Unlike most previous studies, we employed all the 16 radio channels specified by IEEE 802.15 .4 for $2.4 \mathrm{GHz}$ ISM frequency band (channels 11-26) using two different transmit power levels. For the purpose of measuring local weather conditions, each node was integrated with a sensor (SHT75) [12] to measure the ambient temperature and relative humidity. To begin with, we show the temporal variation of signal strength. To find out the role of temperature and humidity on this variation, we study how signal strength correlates with temperature, relative humidity, and absolute humidity. Furthermore, we apply linear regression to explore the magnitude of these effects. We also highlight the differences between channels, the utility of frequency diversity, and the difference between the transmit power levels used. Our findings may be useful for designing algorithms and protocols which are adaptive and robust against the effects of weather. In particular, RSSI-based ranging and localization could benefit from these results.

In summary, our study has the following main contributions:

- We show that changes in weather conditions affect radio signal strength. Temperature seems to be the best explanatory variable for signal strength variation and has a negative, linear effect on signal strength in general, while high relative humidity may have some effect, particularly when temperature is below $0^{\circ} \mathrm{C}$.

- We show that correlation between signal strength and the studied weather variables vary depending on radio channel and link. Applying frequency diversity will alleviate these effects.

- We show that smaller transmit power results in smaller unexplained variation in received signal strength (in most cases) and thus stronger correlation with the studied weather variables.

The rest of this paper is organized as follows. In Section II, 
some related studies in the field are shortly presented. In Section III, we briefly take a closer look at the central variables used in this paper. Experimental measurements performed are described in Section IV. Thereafter, experimental results are presented and analyzed in Section V, followed by a short discussion in Section VI. Finally, we conclude our work in Section VII.

\section{RELATED WORKS}

Temperature has been the main focus in many recent studies dealing with the effects of weather conditions on link quality. For example, Bannister et al. [1] found a linear decrease of $8 \mathrm{~dB}$ in signal strength when temperature rose from $25^{\circ} \mathrm{C}$ to $65^{\circ} \mathrm{C}$ when they used TI CC2420 radio on a Tmote Sky node, both in their outdoor experiment in the Sonoran Desert and in the lab experiment. They also showed the implications this has for communication range, network connectivity, multi-hop data collection, and RSS-based localization. Based on both outdoor and indoor experiments, Boano et al. [5] showed that the increase in temperature decreases both RSSI (Received Signal Strength Indicator) and LQI (Link Quality Indicator). In the outdoor experiment in a wheat field in Govone, Italy, Boano et al. used Tmote Sky nodes. In their indoor experiment, they used both Tmote Sky (CC2420 radio) and MSB430 nodes (CC1020 radio). They also found that the noise floor readings of both platforms decrease with the increase in temperature. Based on their over 10-day deployment of TelosB nodes in a forest garden, Luo et al. [7] showed that both temperature and relative humidity correlate with RSSI. However, based on linear regression, only the effect of temperature on RSSI can be regarded as relatively significant. Wennerström et al. [8] in their half-year experiment near Uppsala, Sweden, showed how variations in meteorological conditions affect IEEE 802.15.4 link performance when using TelosB nodes with CC2420 radio. Particularly, they studied how variations in PRR (Packet Reception Ratio) and RSSI correlate with temperature, absolute humidity, precipitation, and sunlight. Their results show that PRR and RSSI correlate mostly with temperature, while the correlation with other factors is not so clear. They also observed both diurnal and seasonal variation in PRR. In their recent work, Boano et al. [9] studied the impact of temperature on various WSN platforms and radios (CC2420, CC2520) and showed the different effects of temperature on transmitter and receiver nodes. They also showed that the relation between temperature and RSSI is similar with different platforms and can be approximated as a linear function when using platformspecific parameters. Also, Lin et al. [13] found a temporal variation of RSSI (with MICAz nodes) during their 3-day outdoor experiment, in which different transmit power levels were used, but they did not analyze the cause.

Humidity-related issues have also received a lot of attention in the research community. Anastasi et al. [2] found that the transmission range of mica2 sensor nodes (using RFM ChipCon radios) decreases substantially during rain or fog. Contrary to this, based on their measurements conducted in a potato field with the help of Mica2Dot nodes equipped with
Chipcon CC1000 radios, Thelen et al. [4] showed that radio waves propagate better under high humidity conditions, in the presence of rain and at night for example. They attributed this positive impact to changes in the reflection coefficient of the top of the canopy of the potato field. They showed that RSSI values were positively correlated with RH and negatively with temperature, but they focused only on humidity in their analysis. Capsuto and Frolik [3] demonstrated how rain and snowfall, freezing rain and fog, and humidity can significantly affect RSSI, causing large fades or even complete loss of connectivity. They used Chipcon CC2420DK nodes at three different frequencies of $2.4 \mathrm{GHz}$ ISM band in the experiment. Boano et al. [5] also showed that the effect of both thin and thick fog, and rainfall on RSSI is almost negligible. However, the impact of a very heavy rainfall on wireless communication may be significant. Markham et al. [6] conducted a 26-day measurement in the forest of Wytham Woods, near Oxford and a lab experiment, using T-mote Sky nodes with CC2420 radios. They showed that variations in RSSI are due to the presence of water on a node's casing rather than fading caused by rain. They justified their finding by stating that water is capacitively loading the antenna, thereby changing its radiation pattern.

In contrast to previous studies, our experiment was conducted with AT86RF230 radio transceivers. Further, we utilized all the specified 16 radio channels for $2.4 \mathrm{GHz}$ ISM frequency band with two different transmit power levels and used sensors to measure the ambient temperature and relative humidity in each node.

\section{BACKGROUND}

In the following, the related variables used to measure radio signal strength, temperature and humidity are defined.

\section{A. Received Signal Strength Indicator, RSSI}

The well-known basic metrics used to link quality estimation in WSNs are RSSI (Received Signal Strength Indicator), PRR (Packet Reception Ratio), SNR (Signal to Noise Ratio), and LQI (Link Quality Indicator) [14]. Received Signal Strength Indicator (RSSI) is a standard feature built in most radio transceivers typically employed in WSN nodes and indicates the received radio signal power in a particular radio channel. As specified in the IEEE 802.15.4 standard [15], RSSI is computed by averaging RSSI values over 8 symbol periods $(128 \mu \mathrm{s})$. The IEEE 802.15 .4 standard refers to this (average) RSSI as Energy Detection (ED), as also does the AT86RF230 radio transceiver [11].

The ED value of the AT86RF230 radio used [11] is computed by averaging RSSI values over 8 symbol periods $(128 \mu \mathrm{s})$. In AT86RF230, RSSI is a 5-bit register value with $3 \mathrm{~dB}$ resolution, and $\mathrm{ED}$ value is an 8 -bit register value with $1 \mathrm{~dB}$ resolution. The ED value has $84 \mathrm{~dB}$ range and absolute accuracy of $\pm 5 \mathrm{~dB}$. The RF input power in AT86RF230 can be computed as follows:

$$
\mathrm{P}_{\mathrm{RF}}=\text { RSSI_BASE_VAL }+\left(\mathrm{ED} \_L E V E L-1\right)[\mathrm{dBm}],
$$


where RSSI_BASE_VAL $=-91 \mathrm{dBm} \quad$ (RSSI sensitivity) and ED_LEVEL $=1 . .84$. The minimum ED value $($ ED_LEVEL $=0)$ indicates receiver power less than RSSI_BASE_VAL. The formula used to express RSSI as RF input power $[\mathrm{dBm}]$ is radio-specific and usually can be found in the data sheet of the particular manufacturer.

Throughout this paper, the terms RSSI and signal strength will be used to refer to $R F$ input power $P_{R F}[\mathrm{dBm}]$, unless otherwise stated.

\section{B. Relation between temperature and humidity}

The relationship between different weather variables, such as temperature and humidity, can be quite confusing. As it is well known, temperature and humidity are connected to each other either directly or indirectly. For the purpose of analyzing the results, it is of the essence to know their mutual dependence. In the following, we try to emphasize the differences between various humidity definitions and how they are related to temperature and to each other. For clarification, their relationship is also illustrated in Fig. 1.

Humidity is the amount of water vapor, the gaseous state of water, in the air, and is usually invisible. The maximum amount of water vapor in the air depends on air temperature. Absolute humidity $(\mathrm{AH})$ is the water content in the air, i.e., the mass of water vapor included in a particular volume of air, expressed in $\mathrm{g} / \mathrm{m}^{3}$. Saturated humidity $(\mathrm{SH})$ is the maximum amount of water vapor in the air at particular temperature (the blue line in Fig. 1). Relative humidity $(R H)$ defines, in a percent, how much water vapor $(\mathrm{AH})$ is in the air relative to the maximum amount of water vapor $(\mathrm{SH})$ at the same temperature and pressure. Relative humidity of saturated air is $100 \%$. Dew point (temperature) is the temperature to which air must be cooled down in order that water vapor starts to condense into liquid water or ice $(R H=100 \%)$. [16]

Of particular interest is the point wherein water vapor is changing from gaseous state into liquid (condensation) or solid (deposition, $\mathrm{T}<0^{\circ} \mathrm{C}$ ) state. Condensation/deposition starts when humidity increases or temperature falls, reaching the saturation point $(R H=100 \%)$. The condensed water vapor is called either dew (frost when $\mathrm{T}<0^{\circ} \mathrm{C}$ ) or fog (or clouds), depending on whether formed on a solid surface or in the air. [16]

Absolute humidity $A H\left(\mathrm{~g} / \mathrm{m}^{3}\right)$ can be defined, e.g., as a function of temperature and relative humidity as [17]:

$$
A H(t, R H)=216.7 \cdot\left[\frac{\frac{R H}{100 \%} \cdot A \cdot \exp \left(\frac{m \cdot t}{T_{n}+t}\right)}{273.15+t}\right] \text {, }
$$

where $t$ is the actual temperature $\left({ }^{\circ} \mathrm{C}\right), R H$ the actual relative humidity $(\%), m=17.62, T_{n}=243.12^{\circ} \mathrm{C}$, and $A=$ $6.112 \mathrm{hPa}$.

\section{EXPERIMENT OVERVIEW}

In the experiment, we set up a wireless sensor network operating in $2.4 \mathrm{GHz}$ ISM frequency band in a university campus area in western Finland. The WSN measured and

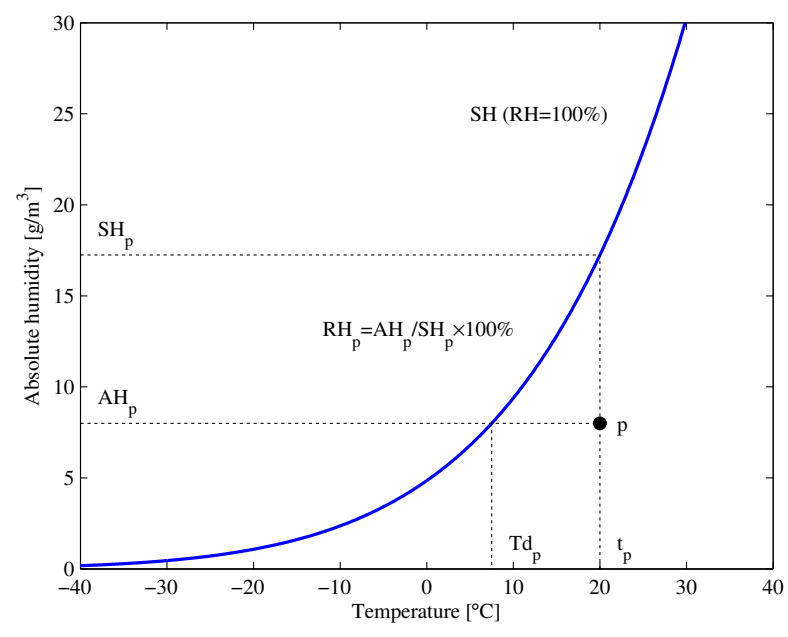

Fig. 1. Relationship between the following weather variables: temperature $(\mathrm{t})$, relative humidity $(\mathrm{RH})$, absolute humidity $(\mathrm{AH})$, saturated humidity $(\mathrm{SH})$ and dew point (Td) (at constant barometric pressure).

collected data related to weather and radio link quality, both in summer and wintertime. This raw data was sent to a server to be further processed and analyzed. In the following, the experiment is described in more detail.

\section{A. WSN Configuration and Deployment}

We used a wireless sensor network operating in $2.4 \mathrm{GHz}$ ISM frequency band in our experiment. The WSN consisted of Atmel ZigBit $2.4 \mathrm{GHz}$ wireless modules (ATZB-24-B0) [10] containing Atmel's ATmega1281V microcontroller [18] and AT86RF230 RF transceiver [11]. Furthermore, the sensor nodes were integrated with a Sensirion's humidity and temperature sensor (SHT75) [12] to measure the ambient temperature and relative humidity. The sensor nodes were powered with two AA-size $3.6 \mathrm{~V}$ primary lithium batteries. The nodes were enclosed with a weatherproof plastic casing, leaving the external antennas and SHT75 sensors outside the casing. A drain valve was added into the bottom of the casing to remove possible moisture or water. The sink node was similar to sensor nodes, without an SHT75 sensor and protective casing, and connected wired to a Raspberry PI. The Raspberry PI had LAN connection for sending raw data from the sink to the server/database.

The equipment used in the experiment consisted of five sensor nodes, one sink node and one Raspberry PI. In addition, there was one server/database. The sensor nodes were attached to five lamp posts with the help of mounting racks. There was one $2.4 \mathrm{GHz}$ node in a single rack. The nodes were at the height of approximately $3 \mathrm{~m}$ (top of the antenna). The sink node and Raspberry PI were inside the university campus, and they were powered by mains current. The network setup can be seen in Fig. 2.

\section{B. Data Collection and Processing}

The sink node broadcasted a link-measurement packet twice in every minute using two different transmit power levels, 


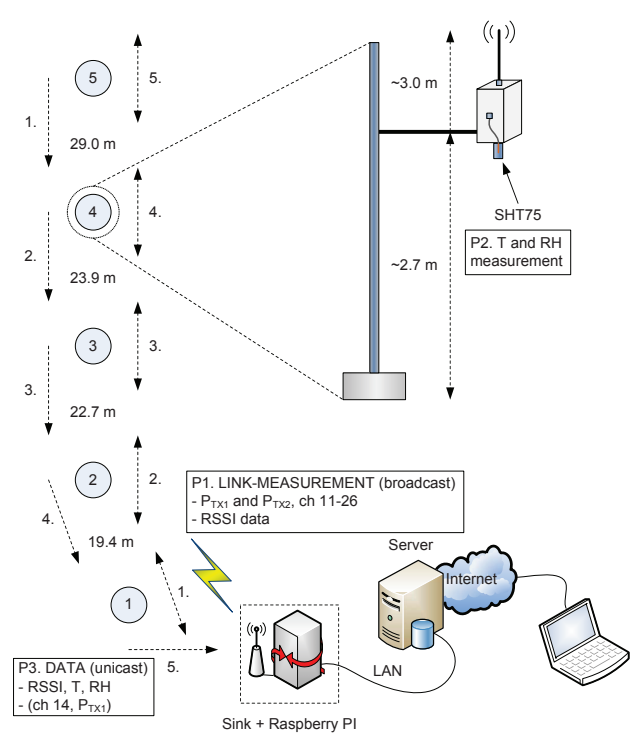

Fig. 2. Measurement network setup (not in scale).

first the maximum transmit power of $+3.0 \mathrm{dBm}\left(\mathrm{P}_{\mathrm{TX} 1}\right)$ and thereafter $-7.2 \mathrm{dBm}\left(\mathrm{P}_{\mathrm{TX} 2}\right)$. Radio channel was changed every minute, thus all the 16 channels $(11-26)$ were rotated in 15 minutes. Sensor nodes receiving the link-measurement packets updated their neighbortables for the particular links (RSSI, etc.), and forwarded the packets in their scheduled time frames. After the link-measurement phase, the nodes measured temperature $(\mathrm{T})$ and relative humidity $(\mathrm{RH})$ readings by using the SHT75 sensor. Thereafter, the sensor nodes sent (unicast) neighbortable and sensor data $(\mathrm{T}, \mathrm{RH})$ to the sink by using predefined static routing (see Fig. 2). The sink forwarded the collected raw data to Raspberry PI, which in turn sent it to the server/database via LAN. Temperature sensor readings (ADC) were converted to temperature values according to [12] and calibrated with each other by using offset values, before saving to the database. Also, RH sensor readings (ADC) were linearized and temperature-compensated according to [12] before saving to the database.

The collected raw data was downloaded from the server to be further processed and analyzed. We used MATLAB [19] for processing, analyzing and presenting data. Both RSSI data (of each channel) and weather data $(\mathrm{T}, \mathrm{RH})$ were averaged over one hour. There were 60 RSSI samples in an hour for each link, i.e., on average $3-4$ samples (for both TX power) for each radio channel. Weather data was measured once in a minute; thus there were 60 samples in an hour for each node. Absolute humidity was also computed; it was based on average temperature and relative humidity values for each node, according to (1).

To mitigate the effects of changes in environmental conditions, effects such as multipath fading, on different radio frequencies, we utilized frequency diversity and computed link RSSI by averaging the RSSI samples collected at different radio channels. The $1 \mathrm{~h}$ average $\mathrm{RSSI}$ for each link $k, \overline{R S S I}_{1 \mathrm{~h}}^{k}$, was computed as

$$
\overline{R S S I}_{1 \mathrm{~h}}^{k}=\frac{1}{n} \sum_{i=1}^{n} R S S I_{1 \mathrm{~h}}^{k, i}[\mathrm{dBm}],
$$

where $i=1 . .16$ (channels $11-26$ specified by IEEE 802.15.4 for $2.4 \mathrm{GHz}$ ISM frequency band). Bardella et al. [20] have shown that exploiting frequency diversity will mitigate the multipath fading effects, which could help us in analyzing the effects of weather conditions.

To calculate statistics (TABLE I), we used the $1 \mathrm{~h}$ average RSSI change of the analyzed links, $\overline{\Delta R S S I}_{1 \mathrm{~h}}$, as follows:

$$
\overline{\Delta R S S I}_{1 \mathrm{~h}}=\frac{1}{8} \sum_{k=1}^{8} \underbrace{\overline{R S S I}_{1 \mathrm{~h}}^{k}-\overline{R S S I}^{k}}_{=\Delta R S S I_{1 \mathrm{~h}}^{k}}[\mathrm{~dB}] \text {, }
$$

where $k$ is the link number, $\overline{R S S I}^{k}$ the average RSSI for link $k$ over the measurement period, and $\triangle R S S I_{1 \mathrm{~h}}^{k}$ the $1 \mathrm{~h}$ RSSI change for link $k$. The links chosen to be analyzed were the ones between the closest neighboring nodes, i.e., the links $1 \leftarrow 2,2 \leftarrow 1,2 \leftarrow 3,3 \leftarrow 2,3 \leftarrow 4,4 \leftarrow 3,4 \leftarrow 5,5 \leftarrow 4$.

As for weather data, we also used $1 \mathrm{~h}$ average temperature and relative humidity of the analyzed links. Absolute humidity was computed based on these average values, applying (1).

\section{EXPERIMENTAL RESUlts}

We performed experiments and gathered data by using our WSN in different seasons. Data from three different periods was chosen, one set in summer and two in winter, to be analyzed here. The representative periods are 1 week in July 2014 (temperature $>0^{\circ} \mathrm{C}$ ), 1 week in December 2013 (temperature $<>0^{\circ} \mathrm{C}$ ), and 3 days in December 2013 (temperature $<0^{\circ} \mathrm{C}$ ). Our aim is to find out how temperature and humidity affect radio signal strength.

\section{A. Temporal Variation of Signal Strength}

When exploring the results from our experiments in both summer and winter, it is evident that signal strength has both short-term (diurnal) and long-term (seasonal/weekly) variation. Interestingly, the variation is notably different in each period. Diurnal variation (day/night) is clearly apparent in summer, whereas seasonal variation is easier to detect in winter and between different seasons (summer/winter). These variations are not random but mainly cyclic, following a certain distinct pattern. For example, in summer, as can be seen in Fig. 3 (a), signal strength falls in the daytime and rises in the nighttime.

Comparing the variation of signal strength with the variation of weather variables in Fig. 3, it is easy to find similarity between them, especially in summer. This suggests that there is a relation between signal strength and particular weather variables. However, the relation in summer seems to be different from that in winter. For example, in summer there is hardly any relation between absolute humidity and signal strength, but in winter below $0^{\circ} \mathrm{C}$ the correlation is quite clear. In contrast, when temperature fluctuates near $0^{\circ} \mathrm{C}$, the relation between weather variables and signal strength is unclear. 

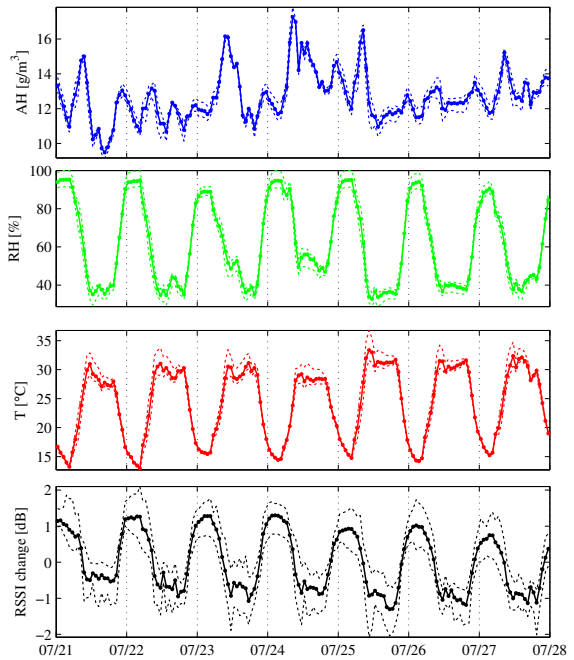

(a) July 2014 (above $0^{\circ} \mathrm{C}$ ).
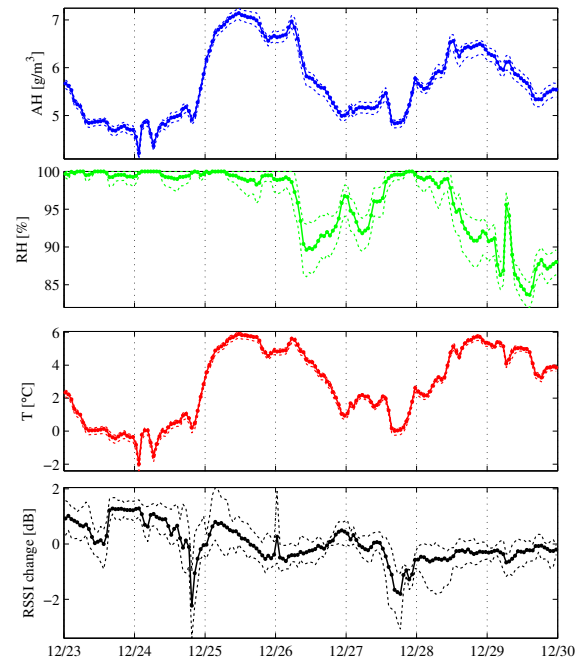

(b) December 2013 (around $0^{\circ} \mathrm{C}$ ).
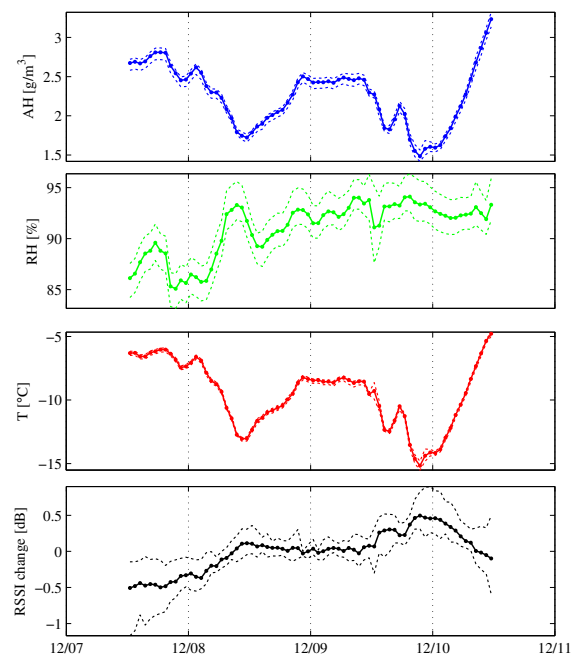

(c) December 2013 (below $0^{\circ} \mathrm{C}$ ).

Fig. 3. RSSI change, temperature (T), relative humidity (RH), and absolute humidity (AH) of the analyzed links (mean, min, max) during three different periods $\left(\mathrm{P}_{\mathrm{TX}}=3.0 \mathrm{dBm}\right)$. $\mathrm{RH}$ data of node 3 is excluded from all computations for December 2013 (around $0^{\circ} \mathrm{C}$ ) due to humidity sensor malfunction.

The behavior of individual links is quite similar, as shown in Fig. 3. This indicates that RSSI variation is mainly caused by changes in weather affecting all the links, rather than by some site-specific reason. However, between individual channels there can be quite large variations in RSSI behavior, both within and between individual links. Probably this results from other factors, such as multipath propagation. By averaging the RSSI samples of different radio channels, we can thus smooth the random and channel-specific variation to better discover the effects of weather conditions.

Nevertheless, our observations confirm the findings reported in the literature (e.g., [1], [8]), which states that signal strength has temporal variation. Probably these variations are mostly due to changes in weather conditions. In the following, we will focus on temperature and humidity to find out their effects on RSSI variation.

\section{B. Effects of Temperature on Signal Strength}

As could already be seen in Fig. 3, there is an obvious relationship between temperature and signal strength. In general, when temperature rises signal strength (RSSI) falls, and vice versa. This indicates negative correlation (dependence) between temperature and signal strength. To get a better understanding of the matter, we computed Pearson correlation coefficient to measure the degree of linear dependence between temperature and RSSI. The results from July are presented in Fig. 4 (a). As can be seen, the correlation varies depending on channel and link. Some link-channel combinations have a very strong correlation, while in some others the correlation is less significant. The difference between the correlation of average RSSI change with temperature (straight black line) and the individual link-channel correlations with temperature is quite clear, indicating the benefits of exploiting frequency diversity. The correlation of the average RSSI change (also channel-specific) is strong, confirming the hypothesis that RSSI correlates negatively with temperature.

The correlation of average RSSI change with temperature is high also while below $0^{\circ} \mathrm{C}$ in December. However, when temperature fluctuates near $0^{\circ} \mathrm{C}$, there is a substantial degradation in correlation. Nevertheless, the negative correlation still holds. The correlation between temperature and RSSI is statistically significant $(p<0.001)$ in each measurement period.

When comparing how RSSI correlates with temperature with two different transmit power levels, we can find some differences. On average, RSSI correlation with temperature is slightly stronger (negatively) when using smaller transmit power $\left(\mathrm{P}_{\mathrm{TX}}=-7.2 \mathrm{dBm}\right)$ compared to when using the maximum transmit power $\left(\mathrm{P}_{\mathrm{TX}}=3.0 \mathrm{dBm}\right)$. This holds true for all the three periods, being emphasized in winter when the deviation is bigger.

To quantify the effect of temperature on RSSI, we plotted both RSSI of individual links and average RSSI change versus temperature and applied simple linear regression, where we used temperature as an explanatory variable for RSSI variation. The results from July are presented in Fig. 4 (b) and 4 (c). As can be seen, temperature has quite a considerable effect on signal strength. A linear, negative trend can be observed for all the links, but there is some variation regarding magnitude of the impact (regression coefficient). As for RSSI change, regression coefficient is -0.127 , i.e., the rise of temperature by $10^{\circ} \mathrm{C}$ decreases RSSI approximately by $1.3 \mathrm{~dB}$. The coefficient of determination $\left(\mathrm{R}^{2}\right)$ is very high (0.93), which implies that in this model RSSI variation can be explained to a high degree by the variation in temperature.

There are no great differences between the different periods regarding the magnitude of the impact. The regression coefficient varies between -0.09 and -0.13 . However, $R^{2}$ decreases during frost, and practically plunges while temperature is 


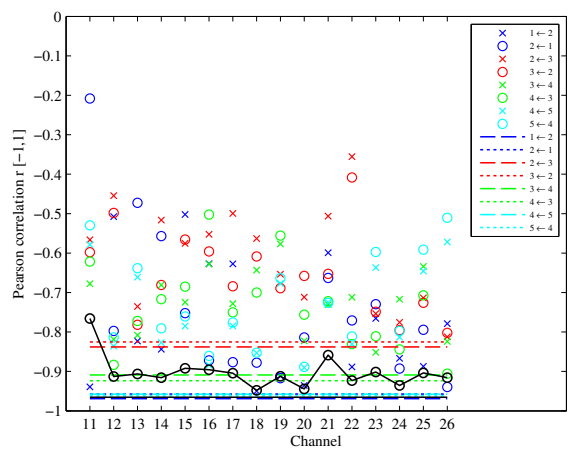

(a)

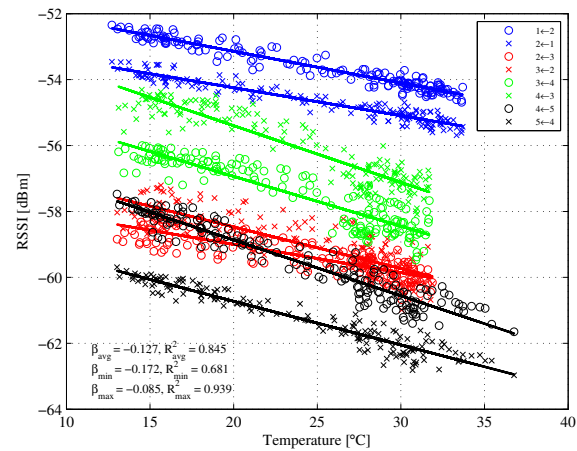

(b)

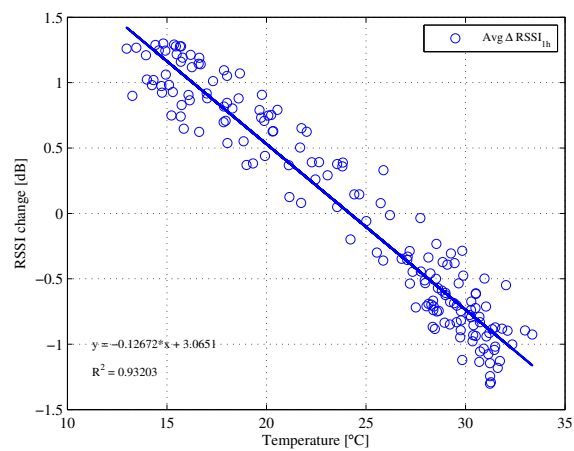

(c)

Fig. 4. Relationship between RSSI/RSSI change and temperature (T) during one week in July $2014\left(\mathrm{P}_{\mathrm{TX}}=3.0 \mathrm{dBm}\right)$. (a) Pearson correlation between RSSI/RSSI change and T, (b) Linear regression of RSSI on T (all links), (c) Linear regression of average RSSI change on T.

around $0^{\circ} \mathrm{C}$. This indicates that when temperature fluctuates around $0^{\circ} \mathrm{C}$, there are some other factors causing large deviations, thus reducing correlation and $\mathrm{R}^{2}$. The regression coefficients are statistically significant $(p<0.001)$ in each measurement period.

As for the magnitude of the effect, the differences between two power levels are not significant. $\mathrm{R}^{2}$ values are somewhat higher with the smaller transmit power $\left(\mathrm{P}_{\mathrm{TX} 2}\right)$, indicating that the smaller transmit power results in smaller unexplained RSSI variation.

The summary of the relationship between temperature and RSSI is presented in TABLE I.

\section{Effects of Humidity on Signal Strength}

Another potential factor affecting signal strength is humidity. As can be seen in Fig. 3, there is also a clear relation between both relative $(\mathrm{RH})$ and absolute $(\mathrm{AH})$ humidity and signal strength in particular times. Relative humidity rises and falls together with RSSI (above $0^{\circ} \mathrm{C}$ ), indicating positive correlation, while the trend of absolute humidity indicates negative correlation (below $0^{\circ} \mathrm{C}$ ). As previously, we computed the Pearson correlation coefficients and simple linear regression for both RH and AH. The summary of the relationship between $\mathrm{RH} / \mathrm{AH}$ and RSSI is presented in TABLE I. The correlation and regression coefficients of $\mathrm{RH}$ to be discussed below are statistically significant $(p<0.01)$ in each measurement period, while those of $\mathrm{AH}$ are statistically significant only for winter periods.

In July, RH has a very high positive correlation (0.95) with RSSI while $\mathrm{AH}$ and RSSI are uncorrelated. $\mathrm{R}^{2}$ in the regression model is also very high for $\mathrm{RH}$, which means that in this model RSSI variation could be explained to a high degree by the variation in RH. As for RSSI change, the regression coefficient of $\mathrm{RH}$ is about 0.03 , which means that the rise of $\mathrm{RH}$ by $10 \%$ increases RSSI approximately by $0.3 \mathrm{~dB}$.

In December, at the temperature below $0^{\circ} \mathrm{C}$, both $\mathrm{RH}$ and $\mathrm{AH}$ have high, almost equally strong but opposite correlation with RSSI. Interestingly, the regression coefficients of both $\mathrm{RH}$ and $\mathrm{AH}$ are about $2-3$ times higher compared to the other period(s). Also $\mathrm{R}^{2}$ is quite significant $(\approx 0.6)$ for both
$\mathrm{RH}$ and $\mathrm{AH}$, although for $\mathrm{RH}$ it is smaller when compared to July.

In the near $0^{\circ} \mathrm{C}$ period, correlation is quite low for both $\mathrm{RH}$ and $\mathrm{AH}$. However, it is still consistent with the other period(s). Due to decreased correlation, it is obvious that $\mathrm{R}^{2}$ in the regression model is also low for both $\mathrm{RH}$ and $\mathrm{AH}$. Therefore, the explanatory powers of the linear regression models are not sufficient.

On average, RSSI correlation with $\mathrm{AH}$ is slightly stronger (negatively) with smaller transmit power in both winter periods, as are the regression coefficients and $\mathrm{R}^{2}$ values. In the case of RH, the differences between the power levels are relatively minor.

\section{Temperature vs. Humidity}

While correlation is a good predictor of a potential causal relationship, it does not imply causation and could be caused by some other factor. The high correlation between the studied weather variables and RSSI both in July (T, RH) and below zero (T, RH, AH) could be partly explained by the high mutual dependence of temperature and humidity, as illustrated in Fig. 5. As can be seen, RH correlates strongly with temperature in July, while AH does so in December. This close relationship between the studied weather variables complicated our attempts to distinguish between the actual impact of temperature and humidity on RSSI. Therefore, we applied multiple linear regression with two explanatory variables to find out both the combined effect and the effect of a particular weather variable while the other variable is taken into account. As previously, to analyze the effects of temperature and humidity on RSSI, we used the average RSSI change, T, RH and $\mathrm{AH}$ of the analyzed links.

As expected, some of the results show high collinearity between temperature and humidity. In July, temperature and $\mathrm{RH}$ are highly collinear (variance inflation factor, VIF is high), while in December below $0{ }^{\circ} \mathrm{C}$, temperature and $\mathrm{AH}$ are collinear. Therefore, it is questionable to use them together in the regression model. Contrary to this, temperature and $\mathrm{AH}$ are not collinear $(\mathrm{VIF}=1.01)$ in July, nor are temperature and $\mathrm{RH}$ $(\mathrm{VIF}=1.38)$ in December when temperature is below $0^{\circ} \mathrm{C}$. 
TABLE I

AVERAGE RSSI CHANGE VS. TEMPERATURE (T) / RELATIVE HUMIDITY (RH) / ABSOLUTE HUMIDITY (AH).

\begin{tabular}{|c|c|c|c|c|c|c|c|c|c|}
\hline $\begin{array}{l}\text { Statistical significance: } \\
* \quad p<0.01 \\
* * \quad p<0.001 \\
\text { otherwise none }\end{array}$ & $\begin{array}{c}\text { July } \\
\left(T>0^{\circ} \mathrm{C}\right) \\
(n=168)\end{array}$ & $\begin{array}{c}\text { RSSI vs. T } \\
\text { December } \\
\left(T<>0^{\circ} \mathrm{C}\right) \\
(n=168)\end{array}$ & $\begin{array}{c}\text { December } \\
\left(T<0^{\circ} \mathrm{C}\right) \\
(n=72)\end{array}$ & $\begin{array}{c}\text { July } \\
\left(T>0^{\circ} \mathrm{C}\right) \\
(n=168)\end{array}$ & $\begin{array}{c}\text { RSSI vs. RH } \\
\text { December } \\
\left(T<>0^{\circ} \mathrm{C}\right) \\
(n=168)\end{array}$ & $\begin{array}{c}\text { December } \\
\left(T<0^{\circ} \mathrm{C}\right) \\
(n=72)\end{array}$ & $\begin{array}{c}\text { July } \\
\left(T>0^{\circ} \mathrm{C}\right) \\
(n=168)\end{array}$ & $\begin{array}{c}\text { RSSI vs. AH } \\
\text { December } \\
\left(T<>0^{\circ} \mathrm{C}\right) \\
(n=168)\end{array}$ & $\begin{array}{c}\text { December } \\
\left(T<0^{\circ} \mathrm{C}\right) \\
(n=72)\end{array}$ \\
\hline \multicolumn{10}{|l|}{ Pearson correlation $(r)$} \\
\hline $\mathrm{P}_{\mathrm{TX} 1}=+3.0 \mathrm{dBm}$ & $-0.965^{* *}$ & $-0.336^{* *}$ & $-0.851^{* *}$ & $0.946^{* *}$ & $0.229^{*}$ & $0.776^{* *}$ & -0.022 & $-0.264^{* *}$ & $-0.779^{* *}$ \\
\hline $\mathrm{P}_{\mathrm{TX} 2}=-7.2 \mathrm{dBm}$ & $-0.973^{* *}$ & $-0.446^{* *}$ & $-0.891^{* *}$ & $0.948^{* *}$ & $0.316^{* *}$ & $0.768^{* *}$ & -0.050 & $-0.353^{* *}$ & $-0.825^{* *}$ \\
\hline \multicolumn{10}{|l|}{ Regression coef. } \\
\hline $\mathrm{P}_{\mathrm{TX} 1}=+3.0 \mathrm{dBm}$ & $-0.127^{* *}$ & $-0.101^{* *}$ & $-0.090^{* *}$ & $0.035^{* *}$ & $0.032^{*}$ & $0.081^{* *}$ & -0.013 & $-0.227^{* *}$ & $-0.539^{* *}$ \\
\hline $\mathrm{P}_{\mathrm{TX} 2}=-7.2 \mathrm{dBm}$ & $-0.113^{* *}$ & $-0.121^{* *}$ & $-0.115^{* *}$ & $0.031^{* *}$ & $0.040^{* *}$ & $0.098^{* *}$ & -0.026 & $-0.276^{* *}$ & $-0.701^{* *}$ \\
\hline \multicolumn{10}{|l|}{$\mathbf{R}^{2}$} \\
\hline $\mathrm{P}_{\mathrm{TX} 1}=+3.0 \mathrm{dBm}$ & 0.932 & 0.113 & 0.724 & 0.895 & 0.053 & 0.602 & 0.000 & 0.069 & 0.607 \\
\hline $\mathrm{P}_{\mathrm{TX} 2}=-7.2 \mathrm{dBm}$ & 0.947 & 0.199 & 0.795 & 0.899 & 0.100 & 0.590 & 0.002 & 0.124 & 0.680 \\
\hline
\end{tabular}

The results from July show that temperature is the dominating factor affecting RSSI. Using $\mathrm{AH}$ as the other explanatory variable together with temperature in the model does not improve the adjusted $R^{2}\left(\overline{\mathrm{R}}^{2}\right)$ in practice. Further, the weight of temperature in the model is significantly higher than that of $\mathrm{AH}$. Therefore, it is sufficient to include only temperature in the regression model.

The situation is different in December below $0^{\circ} \mathrm{C}$. While temperature is the most significant variable $\left(\overline{\mathrm{R}}^{2}=0.72\right.$ for $\left.\mathrm{P}_{\mathrm{TX} 1}\right)$, using of $\mathrm{RH}$ as the other explanatory variable does improve the $\overline{\mathrm{R}}^{2}$ to a relatively large extent $\left(\overline{\mathrm{R}}^{2}=0.87\right)$. This means that both temperature and $\mathrm{RH}$ seem to have effect on RSSI. However, the proportion of temperature in RSSI variation is somewhat higher than that of RH. It is thus reasonable to consider the inclusion of both temperature and $\mathrm{RH}$ in the regression model.

When temperature is around $0^{\circ} \mathrm{C}$ in December, there are other factors apart from temperature or humidity (although probably related to them) causing sudden RSSI variations, as can be seen in Fig. 3. RSSI can experience large variations when temperature fluctuates near $0^{\circ} \mathrm{C}$ and $\mathrm{RH}$ is close to $100 \%$, resulting in low $\mathrm{R}^{2}$ for all the variables. RSSI variation thus cannot be explained with the help of any linear model in this case.

In conclusion, temperature generally seems to be the most significant variable affecting RSSI and could be used in a linear model to explain RSSI change, except in the abovementioned special case. Relative humidity may have some effect on RSSI, particularly in high humidity conditions below $0^{\circ} \mathrm{C}$, where it may be useful to include both temperature and RH in the model.

\section{DisCUSSION}

Our findings confirm the previous results in the literature of the effects of temperature on link quality. Moreover, some $\mathrm{RF}$ transceiver manufacturers mention the temperature dependency in their data sheets [21], [22], [11], thus supporting the results. It has been reported that both output power and receiver sensitivity [21], as well as crystal frequency accuracy (drift) and characteristics of the VCO (Voltage Controlled Oscillator) [21], [22] vary with temperature.

As it was shown, the effect of humidity is more complicated. Additionally, the accuracy of SHT75 sensor decreases substantially during high humidity conditions $(\mathrm{RH}>90 \%)$. It decreases linearly from typical $\pm 1.8 \% \mathrm{RH}$ to $\pm 4.0 \% \mathrm{RH}$ when $\mathrm{RH}$ increases from $90 \%$ to $100 \%$ [12]. Furthermore, [12] states that long term exposure to conditions outside the sensor's normal operating range may temporally offset the $\mathrm{RH}$ signal $(+3 \% \mathrm{RH}$ after $60 \mathrm{~h})$. During both our winter periods, relative humidity is high and mostly over $90 \%$. According to [12], RH can drop drastically due to heavy condensation of water on the sensor surface, which was also observed in our experiment. The foregoing aspects may affect the accuracy of the results in winter.

Particularly problematic is the case when near-zero temperature is combined with high humidity ( $\mathrm{RH}$ close to 100\%). RSSI fluctuation in that period could be due to ice/snow on top of the nodes and antennas melting into liquid water and/or liquid water (due to condensation or rain) freezing into ice. The same kinds of effects of water/ice on link quality are reported, e.g., in [6] and [3]. This indicates that water in liquid or solid state on top of the nodes or antennas may cause unpredictable changes in signal strength. Therefore, temperature and humidity may have indirect effects on RSSI variation through condensation $(\mathrm{RH} \approx 100 \%)$ and freezing of water or melting of ice/snow $\left(\mathrm{T} \approx 0^{\circ} \mathrm{C}\right.$ ).

Regardless, the effects of temperature and humidity on signal strength can have severe implications on different sensor network protocols. Particularly, the accuracy of RSSI-based ranging and localization decreases significantly if the effects of temperature and humidity are ignored. Therefore, temperature and humidity conditions should be taken into account in RSSI-based ranging in order to adapt to prevailing weather conditions. Our results could be used to compensate RSSI variation caused by temperature and humidity, and thus to improve ranging accuracy. Based on the experiment, it could be advisable to use frequency diversity in RSSI-based ranging and localization instead of a single channel. Further, the effects of temperature and humidity have implications on many other 


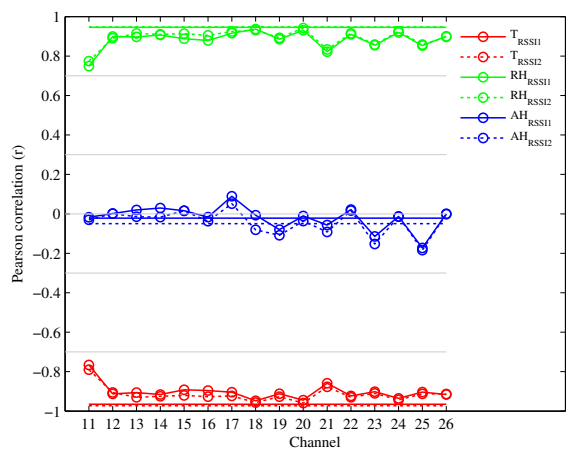

(a) July 2014 (above $0^{\circ} \mathrm{C}$ ).
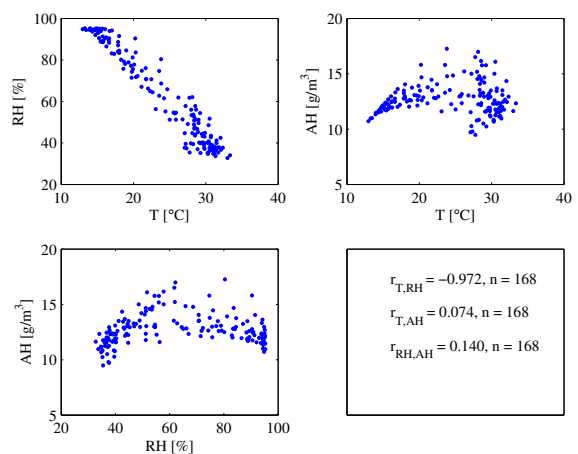

(d) July 2014 (above $0^{\circ} \mathrm{C}$ ).

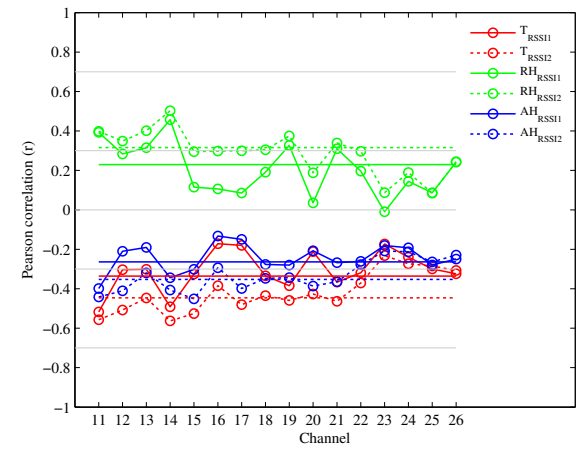

(b) December 2013 (around $0^{\circ} \mathrm{C}$ ).
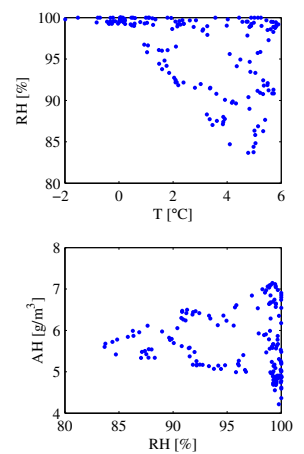

(e) December 2013 (around $0^{\circ} \mathrm{C}$ ).

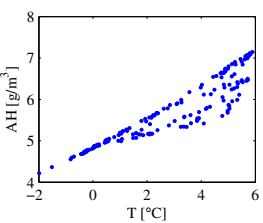

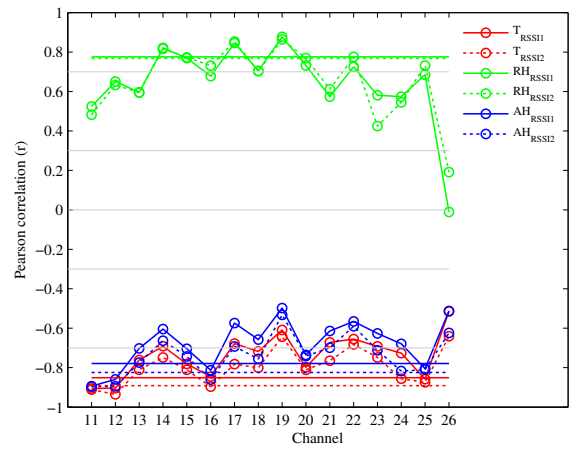

(c) December 2013 (below $0^{\circ} \mathrm{C}$ ).
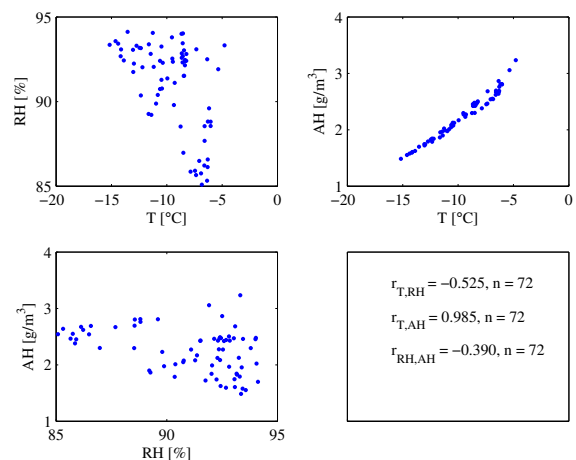

(f) December 2013 (below $0^{\circ} \mathrm{C}$ ).

Fig. 5. Correlation between average RSSI change and weather variables (T, RH, AH) during three different periods (a-c). Correlation between temperature $(\mathrm{T})$, relative humidity $(\mathrm{RH})$, and absolute humidity $(\mathrm{AH})$ during three different periods (d-f).

protocols besides localization. These include protocols related to network connectivity and management, routing, etc.

Weather conditions are not the only reason for RSSI variation. Also HW-related issues and other environmental conditions, such as changes in surroundings (especially in winter), interference, etc. may affect signal strength. The effect of RSSI resolution on the results is also unknown. Furthermore, the measurement period we used was relatively sparse. By using a more dense measurement period as well as applying some filtering method to RSSI readings, it could be possible to achieve more accurate results.

In our future studies, we intend to carry out more experiments and lab measurements to study the effects of temperature and humidity on radio link quality in a controlled environment.

\section{CONCLUSION}

In this paper, the effects of ambient temperature and humidity on radio signal strength of Atmel ZigBit $2.4 \mathrm{GHz}$ wireless modules in outdoor WSNs were explored. Experimental results show that changes in weather conditions affect received signal strength. Temperature seems to have a significant negative influence on signal strength in general, while high relative humidity may have some effect on it, particularly below $0^{\circ} \mathrm{C}$. Further, it was shown that use of frequency diversity can reduce the effects of channel-specific variation, and the difference between the transmit power levels used. Our findings could be useful when designing adaptive, robust algorithms and protocols, such as those related to RSSI-based localization.

\section{REFERENCES}

[1] K. Bannister, G. Giorgetti, and S. Gupta, "Wireless Sensor Networking for "Hot" Applications: Effects of Temperature on Signal Strength, Data Collection and Localization," in The Fifth Workshop on Embedded Networked Sensors (HotEmNets'08), Charlottesville, Virginia, USA June 2008

[2] G. Anastasi, A. Falchi, A. Passarella, M. Conti, and E. Gregori, "Performance Measurements of Motes Sensor Networks," in 7th ACM International Symposium on Modeling, Analysis and Simulation of Wireless and Mobile Systems (MSWiM'04), Venice, Italy, October 2004 pp. 174-181, http://dx.doi.org/10.1145/1023663.1023695.

[3] B. Capsuto and J. Frolik, "A System to Monitor Signal Fade Due to Weather Phenomena for Outdoor Sensor Systems," in Fifth International Conference on Information Processing in Sensor Networks (IPSN 2006), Nashville, TN, USA, April 2006, Demo Abstract.

[4] J. Thelen, D. Goense, and K. Langendoen, "Radio Wave Propagation in Potato Fields," in 1st Workshop on Wireless Network Measurements (WiNMee 2005), Riva del Garda, Trentino, Italy, April 2005.

[5] C. Boano, J. Brown, Z. He, U. Roedig, and T. Voigt, "Low-Power Radio Communication in Industrial Outdoor Deployments: The Impact of Weather Conditions and ATEX-Compliance," in Sensor Applications, Experimentation, and Logistics: First International Conference, SENSAPPEAL 2009, ser. Lecture Notes of the Institute for Computer Sciences, Social Informatics and Telecommunications Engineering, N. Komninos, Ed., vol. 29. Athens, Greece: Springer, September 2009, pp. 159-176, revised Selected Papers, http://dx.doi.org/10.1007/978-3642-11870-8_11.

[6] A. Markham, N. Trigoni, and S. Ellwood, "Effect of Rainfall on Link Quality in an Outdoor Forest Deployment," in International Conference on Wireless Information Networks and Systems (WINSYS 2010), Athens, Greece, July 2010, pp. 1-6. 
[7] J. Luo, X. Xu, and Q. Zhang, "Understanding Link Feature of Wireless Sensor Networks in Outdoor Space: a Measurement Study," in IEEE Global Telecommunications Conference (GLOBECOM 2011), Houston, TX, USA, December 2011, http://dx.doi.org/10.1109/GLOCOM.2011. 6134117.

[8] H. Wennerström, F. Hermans, O. Rensfelt, C. Rohner, and L. Nordén, "A Long-Term Study of Correlations between Meteorological Conditions and 802.15.4 Link Performance," in 2013 IEEE International Conference on Sensing, Communications and Networking (SECON), New Orleans, LA, USA, June 2013, pp. 221-229, http://dx.doi.org/10.1109/SAHCN. 2013.6644981.

[9] C. Boano et al., "Hot Packets: A Systematic Evaluation of the Effect of Temperature on Low Power Wireless Transceivers," in 5th Extreme Conference on Communication (ExtremeCom'13), Thorsmork, Iceland, August 2013.

[10] Atmel, "ZigBit ${ }^{\mathrm{TM}} 2.4 \mathrm{GHz}$ Wireless Modules - ATZB-24-A2/B0 Datasheet," 2009, http://www.atmel.com.

[11] — "Low Power 2.4 GHz Transceiver for ZigBee, IEEE 802.15.4, 6LoWPAN, RF4CE and ISM Applications - AT86RF230 Datasheet,' 2009, http://www.atmel.com.

[12] Sensirion, "Datasheet SHT7x (SHT71, SHT75) - Humidity and Temperature Sensor IC," 2011, Version 5, http://www.sensirion.com.

[13] S. Lin et al., "ATPC: Adaptive Transmission Power Control for Wireless Sensor Networks," in The 4th ACM Conference on Embedded Networked Sensor Systems (ACM SenSys 2006), Boulder, Colorado, USA, Novem- ber 2006, pp. 223-236, http://dx.doi.org/10.1145/1182807.1182830.

[14] N. Baccour et al., "Radio Link Quality Estimation in Wireless Sensor Networks: A Survey," ACM Transactions on Sensor Networks (TOSN), vol. 8, no. 4, September 2012, article 34, http://dx.doi.org/10.1145/ 2240116.2240123 .

[15] IEEE Computer Society, IEEE Std 802.15.4-2003, IEEE, New York, NY, USA, 2003.

[16] Finnish Meteorological Institute, http://ilmatieteenlaitos.fi/.

[17] Sensirion, "Humidity at a Glance - Most Relevant Equations with Sample Code," 2008, Application Note (Version 1.0), http://www.sensirion. com.

[18] Atmel, "Atmel 8-bit AVR Microcontroller with 64K/128K/256K Bytes In-System Programmable Flash," 2006.

[19] The MathWorks, Inc., "MATLAB - The Language of Technical Computing," http://www.mathworks.se/products/matlab/.

[20] A. Bardella, N. Bui, A. Zanella, and M. Zorzi, "An Experimental Study on IEEE 802.15.4 Multichannel Transmission to Improve RSSI-Based Service Performance," in 4th International Workshop on Real-World Wireless Sensor Networks (REALWSN 2010), ser. LNCS 6511, Colombo, Sri Lanka, December 2010, pp. 154-161, http://dx.doi.org/10.1007/9783-642-17520-6 15.

[21] Texas Instruments Inc., "CC2400 - 2.4 GHz Low-Power RF Transceiver," 2008, Data sheet.

[22] —, "CC2420 - 2.4 GHz IEEE 802.15.4 / ZigBee-ready RF Transceiver," 2014, Data sheet. 